\title{
Integrating Life Cícle Aspects Within Product Family Design: An Example for SMEs
}

\author{
H.E. Otto, K.G. Mueller and F. Kimura \\ Dept. of Precision Engineering, The University of Tokyo \\ Bunkyo-ku, Hongo 7-3-1, Tokyo 113-8656, JAPAN \\ tel. $+81-3-5841-6495-$ fax. $+81-3-3812-8849$ \\ e-mail:[otto,kmueller,kimura]@cim.pe.u-tokyo.ac.jp
}

\author{
M. Germani and F. Mandorli \\ Department of Mechanical Engineering, The University of Ancona \\ Via Brecce Bianche, Ancona I-60131, ITALY \\ tel.: +39-71-2204969-fax.:+39-71-2204801 \\ e-mail:germani@popcsi.unian.it,ferro@ied.unipr.it
}

\begin{abstract}
Recent concern for the environment and high competition in globalized markets pose eminent requirements to the producing industry, including small and medium sized enterprises. Envisioned goals of a eco-design combined with a drastical reduction of product development time and increase of customized features of products require new strategies and tools. One solution subject to international investigation is the development of modular products. This approach we enhance by integrating life cycle assessment. Work done in this field was concentrated on developing methods to define either a product's platform architecture or relationships between product modules and documented customer needs within the design analysis of product families. Unfortunately, all of these approaches failed to include a framework to enable also the integration of life cycle assessment. Within the scope of the work described in this paper, we target fundamental problems within the design of product families regarding the integration of life cycle assessment and economic application of information technology, suitable in terms of cost and performance for small and medium sized enterprises. The application field, where first case studies were undertaken and real product models implemented was within the design of parallel-shaft speed reducers.
\end{abstract}

Keywords: product family design, eco design, life cycle inventory parameters, life cycle assessment

\section{INTRODUCTION}

Life cycle assessment (LCA) is becoming increasingly more important as an assessment tool within the design process. LCA may be used in an attempt to minimize certain environmental impacts of a design during the whole life cycle, and as a result weak points and areas requiring special attention of a design are highlighted. The downside of LCA is that a large amount of data is required. The data is difficult to assemble alone due to the volume and even requires periodical updates, which makes LCA relatively expensive. The cost of carrying out an LCA can often not be justified in small and medium sized enterprises (SMEs), since 
savings on the product side cannot be guaranteed to compensate the expenses on the development side.

Realizing that many SMEs specialize on a product, which is produced within a product family, it seems reasonable to prepare an industry specific, parameterized LCA for the product family. The approach taken here is to utilize existing affordable software products and to develop software links in an appropriate manner, resulting in a greatly reduced effort for both the layout of the varied design within the product family and the LCA evaluation.

Since an LCA firstly requires the life cycle inventory (LCI) of the product, the approach described here is to parameterize the LCI using sizing parameters (design parameters) for the product family. A database with LCI data on relevant generic raw- and engineering materials and energy products and a database with site specific manufacturing LCI data are both linked to the simplified product inventory, thus providing a product specific LCI. Actual values for the sizing parameters, which are used to determine the LCI, are extracted from a digital spreadsheet used during the design process.

\section{PRODUCT FAMILY DESIGN AND LCA}

\subsection{Current situation in practice and research}

Today's world wide practice of mass production mostly targeting low product costs determined by high productivity at minimum costs is about to change due to several reasons, as outlined earlier. New approaches to product design and development such as modular product development and eco-design are among the most progressively pursued.

The need for modular product development has increased substantially over the recent years and therefore considerable research attention has been dedicated to solve related problems. Work done in this field was first concentrated on developing methods to define a product's platform architecture [1,2,3]. An approach where a modular product is based on a structure (rules and relations), that is called product platform, on which the designer can assembly different modules developing new product variants to satisfy individual customer requirements. Obviously, a right definition of the product's platform architecture permits an easy configuration of variants (high interchange ability of modules). Other important research issues are the definition of modules and modularity, and the relative identification in an existing product. In several approaches one can find functionbased methods (c.f. [8]) that have been derived from function diagrams originally developed by Pahl and Beitz [6]. Modules, in this case, are defined as physical assemblies of components that have a correspondence with functional groups defined in the functional structure. Analysis of the function diagrams then allows for the evaluation of modularity and the subdivision of modules. To determine relationships between product modules and documented customer needs as described in $[4,9]$ represents yet another approach within the design analysis of product families. An approach that allows to automate the product configuration on the basis of customer requirements.

'Integrating' LCA into the design process is still often seen as simply carrying out LCA in parallel to the design process. The material requirements are extracted from the design manually and entered into an LCA program together with data on the companies manufacturing processes and information on the use and post-use phases. This becomes more difficult with increasing complexity. For LCAs at the 
embodiment and detailing phase of the design process, methods for greater integration (avoiding data redundancy, by reducing the amount of LCI relevant information that is repeatedly determined and input) have been proposed, though non have been implemented in commercial software. They have in common that only the pre-manufacturing and manufacturing phase is assessed, while other phases are neglected.

One trend is the automated extraction of material requirements from CADdrawings, which can be used as an input to LCA programs. The information does not include manufacturing data, which have to be entered manually. A step further is the utilization of a feature technology based CAD approach. Here the geometrical representation of the product is obtained by defining simple geometric objects through a predefined structure, which includes design features (e.g. geometry, dimensions) as well as manufacturing features (e.g. material, machining process). The objects are set in relation to each other to form a part, and the parts are arranged to define a product. Thus the product model includes structured inventory and inventory related information, that can be automatically extracted to determine an overall manufacturing and pre-manufacturing inventory. A specific example can be found in [5]. The disadvantage of this implementation is that it is a 'closed system', thus there is no possibility of interfacing the program to exchange the product's pre-use inventory data with existing LCA software, which would allow a full life cycle assessment. Another example is the method proposed by Birkhofer's group (c.f. [7]), which aims at integrating process and product development by integrating, what they call 'environmental knowledge', into the design process. The main feature of the computer-based system is the interface to product and process development (CAD) and to internal and external environmental information. The environmental information is previous LCA data of materials, machine elements, sub-assemblies, etc. The method is aimed at the embodiment stage of the design process and does not include a systematic approach to include the use-phase of the product.

In summary it may be concluded that current LCA software represent standalone products and research approaches concentrate on specific phases of the life cycle, often eliminating use and post-use phases and only assessing manufacturing and pre-manufacturing phases. These approaches seem to be too inflexible, complex and expensive for especially SME's, where often there is no dedicated LCA team looking at the life cycle optimization of products.

Despite international efforts in research, latest results in areas discussed above are adopted and put into practice by industry quite slowly, and if so, mostly in big enterprises. However, SME industries still remain somewhat isolated ignoring necessity and benefit of these new approaches, though it seems only a matter of time until they too are forced by intensifying global markets and new the environment and natural resources protecting laws and regulations to adapt to current practices of product development methods.

\subsection{Targeted problems and overview}

Within the work of introducing LCA to SMEs as described in this paper, the scope of this first attempt was to provide for an affordable LCA that although being somewhat simplified, is helping to reduce efforts and costs to design environmentally friendly product families. Since SMEs usually specialize on a product that is produced within a product family, an industry specific, parameterized LCA for a particular product family can be realized. 
Since an LCA firstly requires the life cycle inventory (LCI) of the product, the approach described here is to parameterize the LCI using sizing parameters (design parameters) for the product family. A database with LCI data on relevant generic raw- and engineering materials and energy products and a database with site specific manufacturing LCI data can then be both linked to the simplified product inventory, thus providing a product specific LCI. Hereby to accommodate to budget and resources available to the SME, the development of a framework employing easy to handle, inexpensive commercially available hardware platforms and software products was given high priority. To test in how far such an approach can be translated into practice, development and implementation of a fully operating prototype system is targeted.

\section{ECO-DESIGN AND PRODUCT FAMILY DESIGN}

\subsection{Outline of the approach}

Basic requirements - among other things - stemming from the profile of our industrial partner (SME industry) resulted in an approach, providing a framework that allows for transparent, flexible, inexpensive and easy to handle implementation. To design product families and carry out computation of basic life cycle inventory parameters, easy to handle graphical user interfaces are provided to access all central system components (see detailed discussion below), which employ only inexpensive commercially available software that can be used on basic windows NT workstations.

In the next following sections details of methods used in the framework will be discussed together with an outline of the system architecture and tools used for implementation. For evaluation of both the approach and the computer-aided generation of design solutions regarding applicability, performance and future innovative improvements, a first implemented prototype system has been provided for both sides, our research team and our collaborating SME industrial partner.

\subsection{Framework and structures}

Work on the framework was basically aimed at supporting the definition of functional structures by providing well-documented studies of typologies for an application field, subject to investigation. Problems of evaluation of modularity of products can be solved with a functional decomposition of different product typologies as discussed in [4]. The identification of how many common functional groups are present in different products represents the parameter that allows for the definition of commonality in a specific production. Once the modularity is determined, the same functional groups previously identified are then candidates, which become the modules suitable for new products. The correspondence between modules and functions allows for an easy assembly of new configurations to satisfy different customer requirements.

Results of such an analysis are libraries of modules for product designers. However such modules may have different configurations, while performing the same function. Two types of differences, due to technical specifications, shall be mentioned explicitly. Namely, different components dimensions and the use of different components. In the first case, the dimensions can be managed easily with relations and formulas derived from structural constraints, catalogues of standard 
components, etc. In the second case, it is likely to be more difficult, due to the required designer skills, automating the new configuration.

At present, our prototype system supports only the first case. However, in the near future we are going to develop an interface to an integrated KBE system to include and manage the designer's experience.

\subsection{System architecture and prototype implementation}

Currently the implementation of our prototype system supports the configuration of single modules and their assembly. Design parameters are identified with a selection, related to determine the product geometry, which is processed in a digital spreadsheet. This system component in turn is linked to a three-dimensional parametric solid modeling CAD system. To compute a set of life cycle inventory parameters, used to for a basic life cycle assessment, several LCA modules which combine relevant product data and LCI related parameters are integrated in the prototype system. Within this basic framework, a semi-automatic parametric design of product families can be achieved. In a further step, currently underway, the integration of a KBE system and a design knowledge base are prepared (see Figure 1). This shall give the framework and its implementation enough functionality, to organize, relate and process available design parameters, obtained from various sources such as product functionality, customer needs, LCI database, etc. 


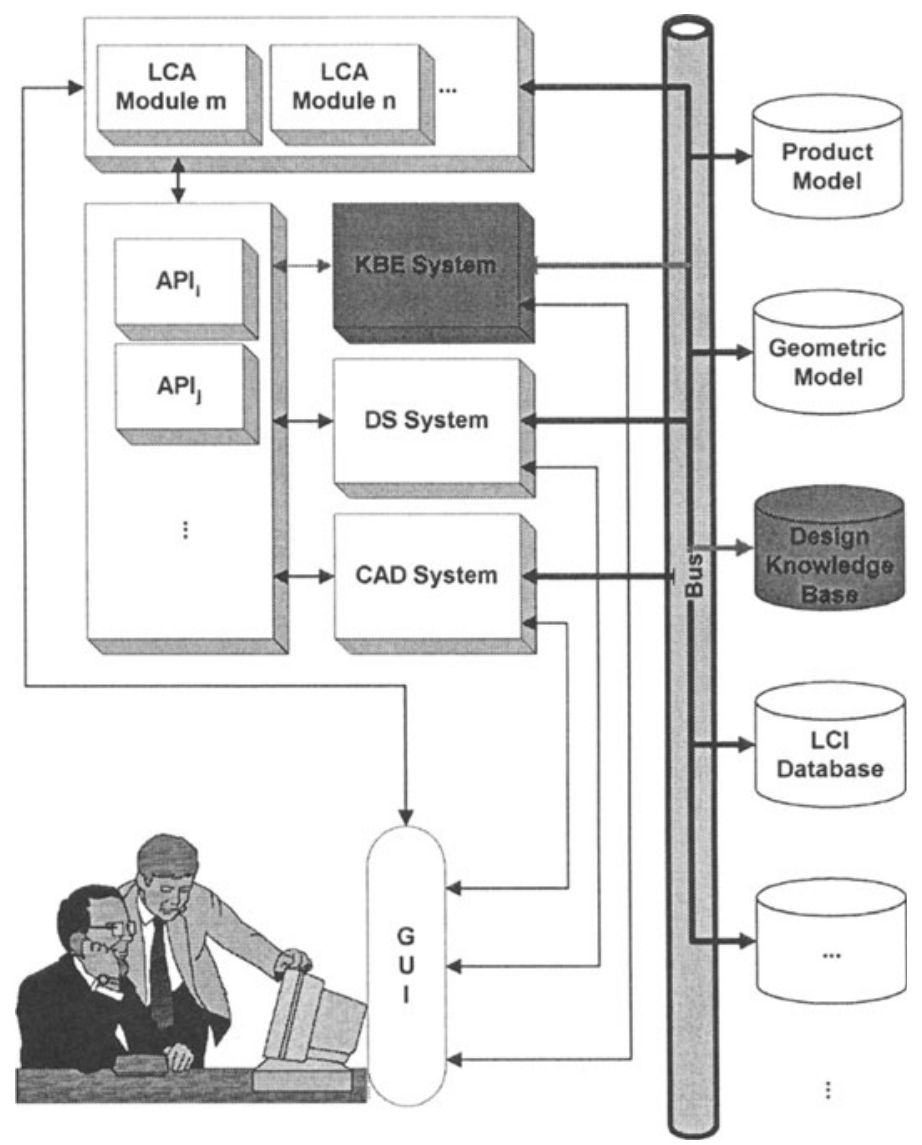

Figure 1 Overview of the system architecture

An important aspect of the system is the link between the digital spreadsheet and the integrated CAD system. Parameters and relations currently implemented within the digital spreadsheet contain all basic design information such as simple design rules, dimensioning relations, dimensions of standard components of a product, etc. During an interactive design session using the implemented prototype system this design information, abstracted as design parameters and relations, turns then together with the user input into the driving force to create as well as control the final overall product dimensions and configurations of module assemblies. Since design parameters are linked to the CAD system as well, further analysis of spatial interference and gaps between components, or studying a better way to execute an assembly, etc. are supported employing the exact geometrical model of a designed part or product. This structured access to the geometrical model is also important for determining some life cycle inventory parameters, when part or product data related to exact geometry such as material volume are required. The open system architecture allows also for an easy interfacing with an FEM system or a CAPP 
system. At the end of a design cycle results of evaluations can be fed back into the system through modifications of design parameters and relations stored in the digital spreadsheet to generate new configurations. In future versions of the system this step of data feedback shall include also complex design knowledge and newly gained experience abstracted as rules of an integrated knowledge base expert system. Eventually the technical documentation (drawings) and bills of material to manage the production processes can be obtained automatically.

Tools and hardware platforms used for implementation and experimental work consisted of windows NT operated workstations and of an object oriented application development environment (VisualStudio) and a digital spreadsheet (Excel) both from Microsoft, a CAD system (SolidEdge) from Unigraphics solutions (UGS), a technical computing environment (MatLab) from MathWorks Inc. and an LCI database.

\section{EXAMPLE}

\subsection{Test data and industrial partner}

We have applied the method described above to the design of a product family of bridge crane speed reducers. In the case study described below subject to examination is a speed reducer for a bridge crane with main requirements of a customer as following: Two different lifting speeds $(0.75 \mathrm{rpm}$ and $7.43 \mathrm{rpm})$ for lifting $63 \mathrm{t}$ with two different powers $3.3 \mathrm{~kW}$ and $33 \mathrm{~kW}$. Additionally the overall dimensions of the entire product should be as small as possible. Our industrial partner within the European Union was an Italian SME (Omme S.P.A.) which is an enterprise working in the field of design and manufacture of custom-made medium and large sized gears and gear trains, respectively. Details of part and product geometry are shown below (cf. Figures 2 and 3 ) as rendered screen dumps created with our implemented prototype system.
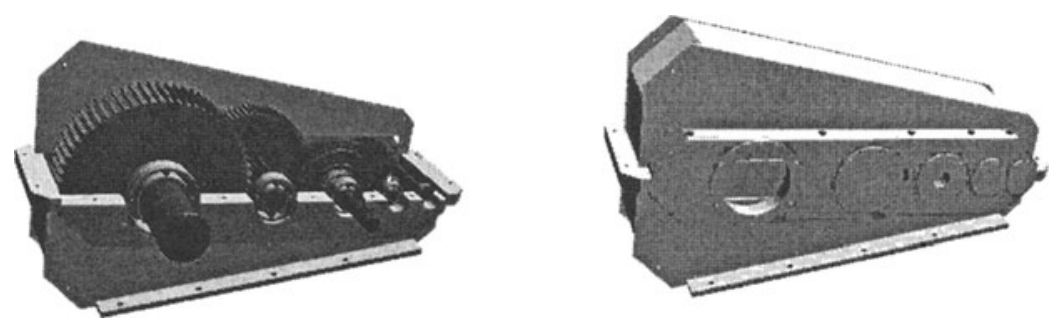

Figure 2 Examples of the housing with and without the gear train 

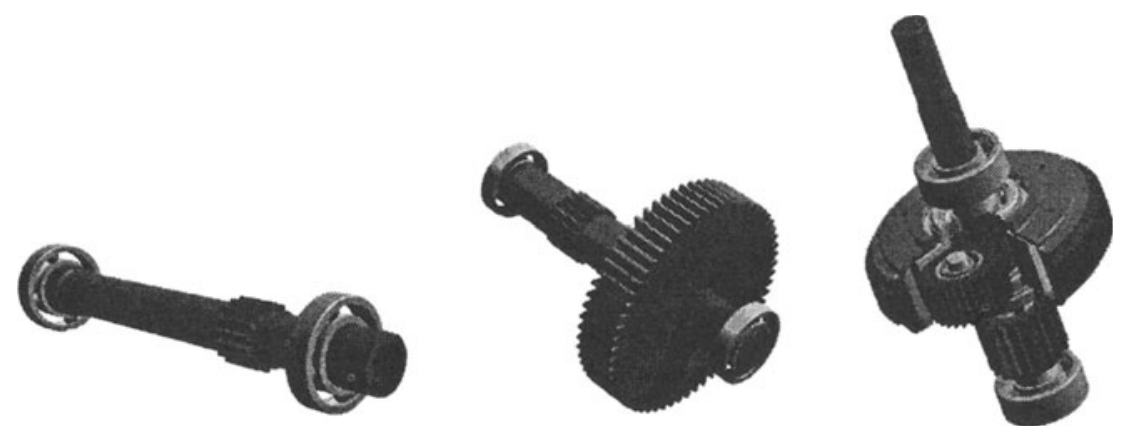

Figure 3 Examples of individual parts and modules

\subsection{Scope and procedure of generated solutions}

The sample outcome of the LCA study of the gearbox is presented in this section. The scope of the LCA is to demonstrate, how with firstly the existing product data, secondly the manufacturing inventory data, thirdly a LCI database on basic energy conversion processes and engineering materials and fourthly the assumptions on the use phase the importance of the different product phases can be compared to each other.

The SME gains from the LCA that weak points of the product are highlighted, but also it is an easy step to perform an assessment of the life cycle costs and these can be minimised once the problem areas are determined. This is an advantageous standpoint, which helps to sell products, especially as many clients are aware that life cycle cost (and the related total cost of ownership) carry significant weight in a sales situation.

The assumptions on the use phase are listed in Table 1 . The average power requirement was determined from the fact that the crane is utilised for loads between $20 \%$ and $100 \%$ of its full load and assuming a symmetrical load distribution the average load would be $60 \%$ of the full load condition and with constant lifting speeds and lifting distances, the power requirement is proportional to the load.

For the purpose of this paper the parts inventory is simplified and summarised in Table 2. The various types of steel used in the gearbox are not distinguished, as the level of detail is not required. The manufacturing inventory could not be determined in time for this paper and therefore had to be neglected.

The sizing parameters required for the LCI are in essence the parameters listed in Tables 1 and 2, the most relevant being the usage characteristics, the efficiency of the gearbox and the parts inventory of the gearbox. These are the parameters that the SME can influence and so optimise the life cycle of its product. 
Table 1: Assumptions on working conditions of gearbox

\begin{tabular}{|l|c|c|}
\hline \multicolumn{1}{|c|}{} & High-Speed & Low-Speed \\
\hline Working hours per year [h] & 2000 & 2000 \\
\hline Operating per working hour & $50 \%$ & $50 \%$ \\
\hline Operation ratio (high and low speed) & $90 \%$ & $10 \%$ \\
\hline Operating time per year [h] & 900 & 100 \\
\hline Design Life [a] & 10 & 10 \\
\hline Avg. power requirement [W] & 19,800 & 1,980 \\
\hline Electric motor efficiency & $94 \%$ & $94 \%$ \\
\hline Gearbox efficiency & $91 \%$ & $87 \%$ \\
\hline Net Energy Input [kWh] & 208,324 & 2,421 \\
\hline
\end{tabular}

Table 2: Simplified summarised parts inventory of gearbox

\begin{tabular}{|c|c|r|}
\hline \hline Part & Material & Mass [kg] \\
\hline Bearings & Steel & 30.35 \\
\hline Gears & Steel & 475.70 \\
\hline Shafts & Steel & 158.50 \\
\hline Housing & Steel & 200.00 \\
\hline & & 864.55 \\
\hline
\end{tabular}

\subsection{Evaluation of results computed}

It can be seen that the low-speed operating conditions only require about $1 \%$ of the net energy required from the grid while the other high-speed operating conditions accounts for $99 \%$. This would indicate that the gearbox efficiency of the low speed condition does not need specific attention, while for the high speed condition a $1 \%$ increase in the gearbox efficiency would result in about a $1 \%$ decrease in total energy requirement.

In Table 3 a selection of LCI data is listed for demonstration purposes. Nevertheless, this simplified LCI data highlights that for most emissions the use phase dominates the life cycle to such an extent, that it can be generally concluded, that the design of heavily used gearbox requires a focus on increasing the efficiency for its predominating operating condition. In this specific case, from the point of view of the SME producing the gearbox, the gearbox efficiency of $91 \%$ for the high-speed condition (cf. Table 1) should be attempted to being increased in order to have the greatest effect on the whole life cycle of the gearbox. From a practical point of view there would be a point in monetary terms where the additional cost to increase the efficiency would be greater than the energy cost saved. 
Table 3: LCI data, for demonstration purposes only (using The Boustead Model, Boustead Consulting, UK)

\begin{tabular}{|l|r|r|r|r|r|r|}
\hline LCl Data & & & & & \\
\hline Resources & Unit & PreUse & Use & PostUse & Total & Use/Total \\
\hline Gross Energy Requirement & $\mathrm{MJ}$ & 66,000 & $2,456,000$ & - & $2,522,000$ & $97 \%$ \\
\hline Water & $\mathrm{t}$ & 24 & 65 & - & 89 & $73 \%$ \\
\hline Air emissions & & & & & & \\
\hline CO2 Equivalent (20 year horizon) & $\mathrm{t}$ & 4.6 & 145.6 & - & 150.2 & $97 \%$ \\
\hline SOx & $\mathrm{t}$ & 0.04 & 1.92 & - & 1.96 & $98 \%$ \\
\hline NOx & $\mathrm{t}$ & 0.00 & 0.88 & - & 0.88 & $100 \%$ \\
\hline Metal & $\mathrm{kg}$ & 0.02 & 1.12 & - & 1.14 & $98 \%$ \\
\hline Hydrogen Chloride (HCl) & $\mathrm{kg}$ & 0.2 & 4.4 & - & 4.6 & $96 \%$ \\
\hline Water Emissions & & & & & & \\
\hline Chem. Oxygen Demand (COD) & $\mathrm{kg}$ & 0.60 & 1.26 & - & 1.86 & $68 \%$ \\
\hline Biochem. Oxygen Demand (BOD) & $\mathrm{kg}$ & 0.02 & 1.13 & & 1.15 & $98 \%$ \\
\hline Lead (Pb) & $\mathrm{g}$ & 0.6 & 0.0 & - & 0.6 & $0 \%$ \\
\hline Solid Emission & & & & & & \\
\hline Mineral Waste & $\mathrm{t}$ & 0.8 & 1.9 & - & 2.7 & $69 \%$ \\
\hline Mixed Industrial Waste & $\mathrm{kg}$ & 30 & 113 & - & 143 & $79 \%$ \\
\hline Slag/Ash & $\mathrm{kg}$ & 280 & 515 & - & 795 & $65 \%$ \\
\hline Inert Chemical Waste & $\mathrm{kg}$ & 7.5 & 0.0 & - & 7.5 & $0 \%$ \\
\hline
\end{tabular}

\section{CONCLUSIONS}

The paper's aim was to demonstrate that low-cost specialized solutions for product family design and life cycle assessment can be developed for SMEs, tailored to the specific needs. The cost are kept to a minimum by using standard inexpensive packages and linking them with tailor made API's.

It was shown that the sizing within the product family was carried out by defining relevant design parameters and the linked software packages can semiautomatically produce solid models, engineering drawings and bills of materials. The bills of materials and the use-phase parameters were used as input parameters for a LCA, which in the example showed the dominance of the use-phase on the life cycle. The most important factor the SME can influence was shown to be the gearbox efficiency in its predominating operating mode (high speed condition).

\section{REFERENCES}

[1] Allen, R. K. and Carlson-Skalak, S. (1998) Defining Product Architecture During Conceptual Design, in: Proc. of 1998 ASME Design Engineering Technical Conference, September 13-16, Atlanta, GA.

[2] Kohlhase, N. and Birkhofer, H. (1996) Development of Modular Structures: The Prerequisite for Successful Modular Products, Journal of Engineering Design, 7, 3, 279-291.

[3] Martin, M. and Ishii, K. (2000) Design for Variety: A Methodology For Developing Product Platform Architectures, in: Proc. of 2000 ASME Design Engineering Technical Conference, September 10-13, Baltimore, MD. 
[4] Mc Adams, D., Stone, R. and Wood, K. (1999) Functional Interdependence and Product Similarity Based on Customers Needs, Research in Engineering Design, 11, $1-19$.

[5] Müller, D. H. and Oestermann, K. (1997) Ökologische und ökonomische Bewertung technischer Produkte mit Hilfe eines featurebasierten Konstruktionssystems, in: Features verbessern die Produktentwicklung: Integration von Prozessketten, VDIGesellschaft Entwicklung, Konstruktion, Vertrieb, VDI-Verlag, Düsseldorf, Germany, 365-380.

[6] Pahl, G. and Beitz, W. (1996) Engineering Design: A Systematic Approach, Springer-Verlag, New York.

[7] Schott, H., Grüner, C., Büttner, K., Dannheim, F. and Birkhofer, H. (1997) Design for environment - computer based product and process development, in: Proc. of the IFIP WG5.3 4th International Seminar on Life-Cycle Engineering, LIFE CYCLE NETWORKS, Berlin, Germany, Chapman \& Hall London.

[8] Stone, B. R., Wood, L. K. and Crawford, H. R. (1998) A heuristic Method to identify Modules from a Functional Description of a Product, in: Proc. of 1998 ASME Design Engineering Technical Conference, September 13-16, Atlanta, GA.

[9] Tichem, M., Andreasen, M.M. and Riitahuhta, A. (1999) Design of Product Families, in: Proc. of Int. Conf. on Engineering Design ICED 99, August 24-26, Munich, Germany, 1039-1042. 